\title{
2D Transition Metal Carbides (MXenes) for Carbon Capture
}

Persson, Ingemar; Halim, Joseph; Lind, Hans; Hansen, Thomas W.; Wagner, Jakob B.; Näslund, LarsÅke; Darakchieva, Vanya; Palisaitis, Justinas; Rosen, Johanna; Persson, Per O.Å.

Published in:

Advanced Materials

Link to article, DOI:

10.1002/adma.201805472

Publication date:

2019

Document Version

Peer reviewed version

Link back to DTU Orbit

Citation (APA):

Persson, I., Halim, J., Lind, H., Hansen, T. W., Wagner, J. B., Näslund, L-Å., Darakchieva, V., Palisaitis, J., Rosen, J., \& Persson, P. O. A. (2019). 2D Transition Metal Carbides (MXenes) for Carbon Capture. Advanced Materials, 31(2), [e1805472]. https://doi.org/10.1002/adma.201805472

\section{General rights}

Copyright and moral rights for the publications made accessible in the public portal are retained by the authors and/or other copyright owners and it is a condition of accessing publications that users recognise and abide by the legal requirements associated with these rights.

- Users may download and print one copy of any publication from the public portal for the purpose of private study or research.

- You may not further distribute the material or use it for any profit-making activity or commercial gain

- You may freely distribute the URL identifying the publication in the public portal 


\section{D transition metal carbides (MXenes) for carbon capture}

Ingemar Persson, ${ }^{* 1}$ Joseph Halim, ${ }^{1}$ Hans Lind, ${ }^{1}$ Thomas W. Hansen ${ }^{2}$, Jakob B. Wagner, ${ }^{2}$ LarsÅke Näslund, ${ }^{1}$ Vanya Darakchieva, ${ }^{3}$ Justinas Palisaitis, ${ }^{1}$ Johanna Rosen ${ }^{1}$ and Per O. Å. Persson ${ }^{1}$

${ }^{1}$ Thin Film Physics Division, Department of Physics, Chemistry and Biology (IFM), Linköping University, SE-581 83 Linköping, Sweden

${ }^{2}$ Center for Electron Nanoscopy, DTU Danchip/CEN, DK-2800, Kgs. Lyngby, Denmark

${ }^{3}$ Terahertz Materials Analysis Center (THeMAC), Department of Physics Chemistry and Biology (IFM), Linköping University, SE-581 83 Linköping, Sweden

\section{Keywords}

MXene, surface terminations, carbon capture, environmental TEM

Global warming caused by burning of fossil fuels is indisputably one of mankind's greatest challenges in the twenty-first century. To reduce the ever-increasing $\mathrm{CO}_{2}$ emissions released into the atmosphere, dry solid adsorbents with large surface to volume ratio such as carbonaceous materials, zeolites, and metal organic frameworks have emerged as promising material candidates for capturing $\mathrm{CO}_{2}$. However, challenges remain because of limited $\mathrm{CO}_{2} / \mathrm{N}_{2}$ selectivity and long-term stability. The effective adsorption of $\mathrm{CO}_{2}$ gas $\left(\sim 12 \mathrm{~mol} \mathrm{~kg} \mathrm{~kg}^{-1}\right)$ on individual sheets of two-dimensional (2D) transition metal carbides (referred to as MXenes) is reported here. It is shown that exposure to $\mathrm{N}_{2}$ gas results in no adsorption, consistent with firstprinciple calculations. The adsorption efficiency combined with the $\mathrm{CO}_{2} / \mathrm{N}_{2}$ selectivity, together with a chemical and thermal stability, identifies the archetype $\mathrm{Ti}_{3} \mathrm{C}_{2} \mathrm{MXene}$ as a new material for carbon capture (CC) applications. 
In the search for new solid adsorbents that can capture carbon dioxide by physical and chemical adsorption, focus is turned to materials with high surface area including carbonaceous materials, ${ }^{[1]}$ zeolites, ${ }^{[2]}$ metal organic frameworks, ${ }^{[3]}$ and 2D materials such as functionalized graphene oxide. ${ }^{[4]}$ Despite several advantages (moles adsorbed per mass unit, low production cost), they are challenged by poor selectivity for $\mathrm{CO}_{2}$ over $\mathrm{N}_{2}$ and robustness in terms of lifetime and multicycle durability. ${ }^{[5]}$ MXenes are a recent addition to the family of $2 \mathrm{D}$ materials and have emerged with superior properties and performance in terms of stability, ${ }^{[6]}$ electrochemical charge storage, ${ }^{[7,8]}$ electromagnetic interference shielding, ${ }^{[9]}$ filtering, ${ }^{[10]}$ and a range of additional applications. ${ }^{[8]}$

They constitute a large and growing family of $2 \mathrm{D}$ materials, ${ }^{[11,12]}$ that are obtained from the laminated $M_{n+1} A X_{n}(M A X)$ phases ( $M$ is a transition metal, $A$ is a group A element -mostly group 13 and 14 - and $\mathrm{X}$ is $\mathrm{C}$ and/or N) ${ }^{[13]}$ by chemical etching of the atomically thin A element layers that separate sheets of $M_{n+1} X_{n}$. As the A-element is removed, the MXene surfaces are immediately functionalized by surface terminating species, $T_{\mathrm{x}} \cdot{ }^{[6,14]}$ Hence the proper MXene formula is $\mathrm{M}_{\mathrm{n}+1} \mathrm{X}_{\mathrm{n}} \mathrm{T}_{\mathrm{x}}$. Accordingly, the MXene properties can be tuned through structure, intrinsic composition, and surface terminations. The structure is inherited from the parent MAX phase (hexagonal, space group $\mathrm{P}_{3} / \mathrm{mmc}$ ) but compositional tuning display an extraordinary toolbox for property tuning through MXenes based on single $\mathrm{M}$ and $\mathrm{X}$ elements, as well as alloys on both $\mathrm{M}$ and $\mathrm{X} .{ }^{[12,15]}$ In addition, there are reports on MXenes forming out-of-plane ${ }^{[16]}$ and in-plane ${ }^{[17]}$ double-M elemental ordering, as well as vacancy-ordered structures. ${ }^{[18,19]}$ Manipulation of the surface terminations constitute the final and most powerful variable for property tuning. [20] Despite several theoretical investigations, [21,22,23] non-inherent terminations have remained experimentally unexplored. Currently, the MXene preparation dictates that $\mathrm{T}_{\mathrm{x}}$ is inherent to the etchant and predominantly a combination of $\mathrm{O}$ and $\mathrm{F}$, where also $\mathrm{OH}$ has been considered as a minor ${ }^{[24]}$ or even negligible contribution. ${ }^{[25]}$

In the area of $\mathrm{CC}$, MXenes are predicted to be highly efficient for capturing $\mathrm{CO}_{2}$, enabling capture of 2-8 mol $\mathrm{CO}_{2} \mathrm{~kg}^{-1}$. ${ }^{[21,22]}$ However, the MXene surfaces were assumed to be termination free, an experimentally unrealistic starting point, given the current wet-chemical preparation routes for MXenes. To unlock the MXene potential for non-inherent terminations or adsorption of other molecules, such as $\mathrm{CO}_{2}$, we have subjected the archetype $\mathrm{Ti}_{3} \mathrm{C}_{2} \mathrm{~T}_{\mathrm{x}} \mathrm{MXene}$ to a novel approach. Using in situ environmental transmission electron microscopy (ETEM), single $\mathrm{Ti}_{3} \mathrm{C}_{2} \mathrm{~T}_{\mathrm{x}}$ sheets were subjected to an initial high temperature treatment to desorb $\mathrm{F},{ }^{[25]}$ 
and a subsequent $\mathrm{H}_{2}$ exposure to remove the persistent $\mathrm{O}$ from the surfaces. The thereafter termination-depleted MXene was subsequently exposed to $\mathrm{CO}_{2}$ gas, resulting in the first MXene to be terminated by a non-inherent molecule. Additionally, termination-depleted MXene surfaces were exposed to $\mathrm{N}_{2}$ gas after which no $\mathrm{N}$ adsorption was observed, consequently identifying $\mathrm{Ti}_{3} \mathrm{C}_{2}$ as highly efficient also in terms of $\mathrm{CO}_{2} / \mathrm{N}_{2}$ selectivity.

Figure 1a-d represent a cross-section schematic that illustrates, from left to right, the processing that was applied to the $\mathrm{Ti}_{3} \mathrm{C}_{2} \mathrm{~T}_{\mathrm{x}}$ MXene in order to reduce the surface terminating species followed by the adsorption of $\mathrm{CO}_{2}$. Figure 1a describes an as-prepared single $\mathrm{Ti}_{3} \mathrm{C}_{2} \mathrm{~T}_{\mathrm{x}}$ flake terminated by a disordered mixture $(x>2)$, predominantly consisting of $\mathrm{O}$ and $\mathrm{F} .{ }^{[244,25]}$ Figure $1 \mathrm{~b}$ shows how an initial thermal treatment of the sheet at $650^{\circ} \mathrm{C}$ for $12 \mathrm{~h}$ inside the ETEM at a pressure of $10^{-6}$ mbar results in the desorption of $\mathrm{F}$ (see supplementary information Fig. S1), in line with previous investigations. ${ }^{[25]}$ In response to the desorbed $\mathrm{F}$, the remaining $\mathrm{O}$ rearranges itself to cover the surface at the thermodynamically preferred fcc-site (Fig. 1b). ${ }^{[26]}$ With F depleted and only $\mathrm{O}$ remaining, the MXene is locally termination-free $(\mathrm{x}<2)$. Following this initial thermal procedure, Fig. 1c schematically illustrates the subsequent introduction of 8 mbar $\mathrm{H}_{2}$ local gas flow parallel to the sample surface during heating to $700{ }^{\circ} \mathrm{C}$ for $0.5 \mathrm{~h}$, leading to $\mathrm{O}$ depletion. Finally, as shown in Fig. 1d, $3.5 \mathrm{mbar} \mathrm{CO}_{2}$ is introduced at $100{ }^{\circ} \mathrm{C}$ and adsorbed on the depleted surface. The TEM images presented in Fig. 1e-h demonstrate the single MXene flake structure throughout the in situ process, with e) showing the as prepared structure, f) after heating in vacuum, g) after exposure to $\mathrm{H}_{2}$, and $\left.\mathrm{g}\right)$ after exposure to $\mathrm{CO}_{2}\left(100{ }^{\circ} \mathrm{C}\right.$ was chosen to avoid residual hydrocarbon adsorption on the single sheet). Note that the figures and in particular Fig. 1h, are not astigmatic as determined by fast fourier transform (FFT) (presented in supplementary information, Fig. S2), hence the apparent structure is a result of ordering of the $\mathrm{CO}_{2}$ molecules on the surface.

During the heating and gas exposure experiments inside the ETEM, the changes in the $\mathrm{Ti}_{3} \mathrm{C}_{2} \mathrm{~T}_{\mathrm{x}}$ surface chemistry and structure were monitored by electron energy-loss spectroscopy (EELS) and electron diffraction (ED). Residual gas analysis (RGA) was employed to monitor reaction products originating from the interactions of the applied gas and the MXene surface species (shown in the supplementary information, Fig. S3). Following vacuum heating to remove the F terminations, Fig. 2a-c show the EELS spectra acquired from single flakes after 8 mbar $\mathrm{H}_{2}$ exposure for $1 \mathrm{~h}$ intervals at RT, 500, 700, and $750{ }^{\circ} \mathrm{C}$ for $\mathrm{C}-\mathrm{K}$, Ti-L, and $\mathrm{O}-\mathrm{K}$ respectively. The shape of the C-K edge in Fig. 2a confirms that the chemical environment of $\mathrm{C}$ remains 
constant throughout the experiment. A gradual chemical shift up to $-1 \mathrm{eV}$ is observed for Ti- $\mathrm{L}_{3}$ and $\mathrm{Ti}-\mathrm{L}_{2}$ above RT that indicates a decrease of O-terminations (caused by reduced charge transfer from Ti to O). ${ }^{[27]}$ The $\mathrm{O}-\mathrm{K}$ edge is displayed in Fig. $2 \mathrm{c}$, and a $-2 \mathrm{eV}$ energy shift is observed above RT. A plausible cause of the negative shift of O-K is $\mathrm{H}$ saturation of the surface. Most importantly, the integrated intensity of the O-K edge, corresponding to remaining surface terminations, is being reduced with increased temperature. EELS quantification of the Ti:O ratio yield 3:2.1 at $\mathrm{RT}, 3: 1.4$ at $500{ }^{\circ} \mathrm{C}, 3: 1.2$ at $700{ }^{\circ} \mathrm{C}$, and 3: 0.6 at $750{ }^{\circ} \mathrm{C}$. The chemical shifts of Ti-L and O-K in combination with the loss of O, clearly describes a decrease of Oterminations caused by active $\mathrm{H}$ on the $\mathrm{Ti}_{3} \mathrm{C}_{2}$ surface. The apparently depleted surface can also be comprehended from Fig. 1g, which exhibits a clean appearance. Figure $2 \mathrm{~d}$ presents the corresponding ED patterns that verify the preservation of the MXene structure up to $700{ }^{\circ} \mathrm{C}$. At $750{ }^{\circ} \mathrm{C}$ the $\mathrm{ED}$ patterns confirm the formation of $\mathrm{TiC}$ nanoplatelets ${ }^{[28]}$ with similar lattice spacing compared to $\mathrm{Ti}_{3} \mathrm{C}_{2}$ (see supplemental information, Fig. S2). During the MXene exposure to $\mathrm{H}_{2}$, RGA detected the formation of $\mathrm{H}_{2} \mathrm{O}$ from $500{ }^{\circ} \mathrm{C}$, which is increasing slightly with temperature (see supplementary information, Fig. S3). The formation of $\mathrm{H}_{2} \mathrm{O}$ at elevated temperatures correlates well with O-termination depletion which is expected with $\mathrm{H}_{2}$ reacting with surface bound $\mathrm{O}$.

The results show that $\mathrm{H}_{2}$ exposure at elevated temperatures is a facile route for removing $\mathrm{O}$ terminations. Consequently, experiments that probe the fundamental MXene properties are attainable. Because of the low pressure (mbar range) of the applied $\mathrm{H}_{2}$ gas during the limited time frames, complete removal of $\mathrm{O}$ was not achieved in these in situ experiments. Further depletion is proposed to be attainable at higher $\mathrm{H}_{2}$ pressures that are not possible in the ETEM. The high temperature vacuum heating and $\mathrm{H}_{2}$ processes also demonstrate the robust nature of the employed $\mathrm{Ti}_{3} \mathrm{C}_{2} \mathrm{~T}_{\mathrm{x}}$.

Figure 2e-g presents EELS spectra for the C-K, Ti-L, and O-K edges, respectively, obtained from single MXene flakes after heat treatment and $\mathrm{H}_{2}$ exposure at $650^{\circ} \mathrm{C}$ followed by exposure of 3.5 mbar $\mathrm{CO}_{2}$ for $0.5 \mathrm{~h}$ at $100{ }^{\circ} \mathrm{C}$. The $\mathrm{C}-\mathrm{K}$ edge integrated intensity and fine structure radically changes upon $\mathrm{CO}_{2}$ exposure. In particular, the second broad peak centered at $293 \mathrm{eV}$ exhibits a considerable increase in intensity. The Ti- $\mathrm{L}_{3,2}$ edge does not reveal significant fine structure changes (symmetry and bonds remain similar after adsorption), though both the Ti- $\mathrm{L}_{3}$ and Ti- $\mathrm{L}_{2}$ (particularly the Ti- $\mathrm{L}_{2}$ ) edges experience apparent positive chemical shifts. This is indicative of the formation of a chemical bond between the $\mathrm{Ti}$ and adsorbed $\mathrm{CO}_{2}$, resulting in 
charge transfer from the $\mathrm{Ti}$ to the $\mathrm{CO}_{2}$. Figure $2 \mathrm{~g}$ displays a substantial increase in the $\mathrm{O}-\mathrm{K}$ edge intensity. EELS quantification of the sheet stoichiometries before and after $\mathrm{CO}_{2}$ exposure yields a Ti:C:O ratio of 3:2:1.45 and 3:4.3:5.2, respectively, which approximately corresponds to one adsorbed $\mathrm{CO}_{2}$ molecule per $\mathrm{M}$ surface atom. This is equivalent to an uptake capacity of $12 \mathrm{~mol} \mathrm{~kg}^{-1}$ or a relative increase by $52.7 \mathrm{wt} . \%$.

Competing materials for carbon capture include Mg-MOF-74 ${ }^{[29]}$ and Zeolite X13 ${ }^{[30]}$ where $\mathrm{Mg}-\mathrm{MOF}-74$ is reported to adsorb $\mathrm{CO}_{2}$ equivalent to a weight increase of $35 \mathrm{wt} . \%$ at 1 bar (313 K). ${ }^{[29]}$ Zeolite X13 is the benchmark material because of its low cost and a capacity of $22 \mathrm{wt} . \%$ at 1 bar $(298 \mathrm{~K})^{[30]}$ (Details in Table S1). Furthermore, regeneration of zeolite X13 has been reported to be more cost-effective from a long-term perspective than Mg-MOF-74 despite the capacity difference. On that note, MXenes are chemically and thermally stable (as verified here) and therefore exhibit a high potential for regeneration. Thus, the initial assessment of single $\mathrm{Ti}_{3} \mathrm{C}_{2}$ sheets yields that MXene is on par with, or even outperforms, current $\mathrm{CO}_{2}$ adsorbents. $[1,2,3,29,30]$

The potential for MXenes for carbon capture can be further stressed by employing $\mathrm{Ti}_{2} \mathrm{C}$ as an example. Should this MXene adsorb the same amount of $\mathrm{CO}_{2}$ per surface $\mathrm{M}$ element, the weight increase would be an astonishing $80 \mathrm{wt} . \%$. Finally, it is straightforward to alloy most MXenes with a few atomic percent of various metals that can increase the catalytic activity in conversion mechanisms.

Figure $2 \mathrm{~h}$ presents the corresponding ED patterns acquired before and after $\mathrm{CO}_{2}$ exposure, respectively, again confirming the preservation of the MXene structure. The final structure after $\mathrm{CO}_{2}$ exposure as shown in Fig. $1 \mathrm{~h}$ exhibit a streaked surface and this is proposed to originate from the alignment of $\mathrm{CO}_{2}$ molecules on the MXene surface. As demonstrated above, $\mathrm{CO}_{2}$ exposure of the O-depleted MXene immediately leads to complete $\mathrm{CO}_{2}$ saturation of the surface while the $2 \mathrm{D}$ structure is maintained. Complementary to the $\mathrm{CO}_{2}$ exposure, the $\mathrm{H}_{2}$ exposed surfaces were additionally subjected to $\mathrm{N}_{2}$ exposure. During these experiments no adsorbed $\mathrm{N}_{2}$ was detected, indicating that termination-free $\mathrm{Ti}_{3} \mathrm{C}_{2}$ is highly selective between $\mathrm{CO}_{2}$ and $\mathrm{N}_{2}$. This result is explained by our calculations based on density functional theory (DFT) suggesting an endothermic reaction for $\mathrm{N}_{2}$ dissociation combined with formation of $\mathrm{N}$-terminated MXene (see supporting information for details). Altogether, owing to the adsorption efficiency, the selectivity and the large surface area per mass unit, $\mathrm{Ti}_{3} \mathrm{C}_{2}$ MXene is shown to be a highly 
efficient material for carbon capture, which constitute an entirely new, and extremely promising, application for MXenes.

In summary, in this manuscript we report two fundamental findings: 1) We have identified a route for termination of (adsorption on) $\mathrm{Ti}_{3} \mathrm{C}_{2}$ MXene by non-inherent species through a combination of heating and $\mathrm{H}_{2}$ exposure that depleted the $\mathrm{Ti}_{3} \mathrm{C}_{2}$ surfaces from terminating species. It is postulated that terminations on other MXenes can also be depleted in the same fashion. 2) We have identified single sheets of $\mathrm{Ti}_{3} \mathrm{C}_{2}$ MXene as a solid adsorbent for carbon capture applications. Following the surface depletion, a subsequent exposure to $\mathrm{CO}_{2}$, resulted in the adsorption of $\sim 12 \mathrm{~mol} \mathrm{~kg}^{-1} \mathrm{CO}_{2}$ on the $\mathrm{Ti}_{3} \mathrm{C}_{2} \mathrm{~T}_{\mathrm{x}}$, a result that is on par with or even outperforms state of the art $\mathrm{CO}_{2}$ adsorbents at low pressures. It is proposed that, even higher loadings are attainable following $\mathrm{H}_{2}$ and $\mathrm{CO}_{2}$ exposure at higher pressures than presently obtained in the ETEM. We also found that the depleted MXene showed no affinity for $\mathrm{N}_{2}$ gas, consistent with our calculations, making the sheets highly selective towards $\mathrm{CO}_{2}$ over $\mathrm{N}_{2}$. Furthermore, a high stability of the MXene throughout the experiments was demonstrated, in line with previous findings. It is proposed that this may be useful in the subsequent conversion of the captured $\mathrm{CO}_{2}$ and for recycling of the same material in lines with a circular economy. The here presented results enables MXenes as an exceptional and robust breakthrough material for carbon capture. 

a) As prepared
b) Heat treatment
c) $\mathrm{H}_{2}$ exposure
d) $\mathrm{CO}_{2}$ exposure

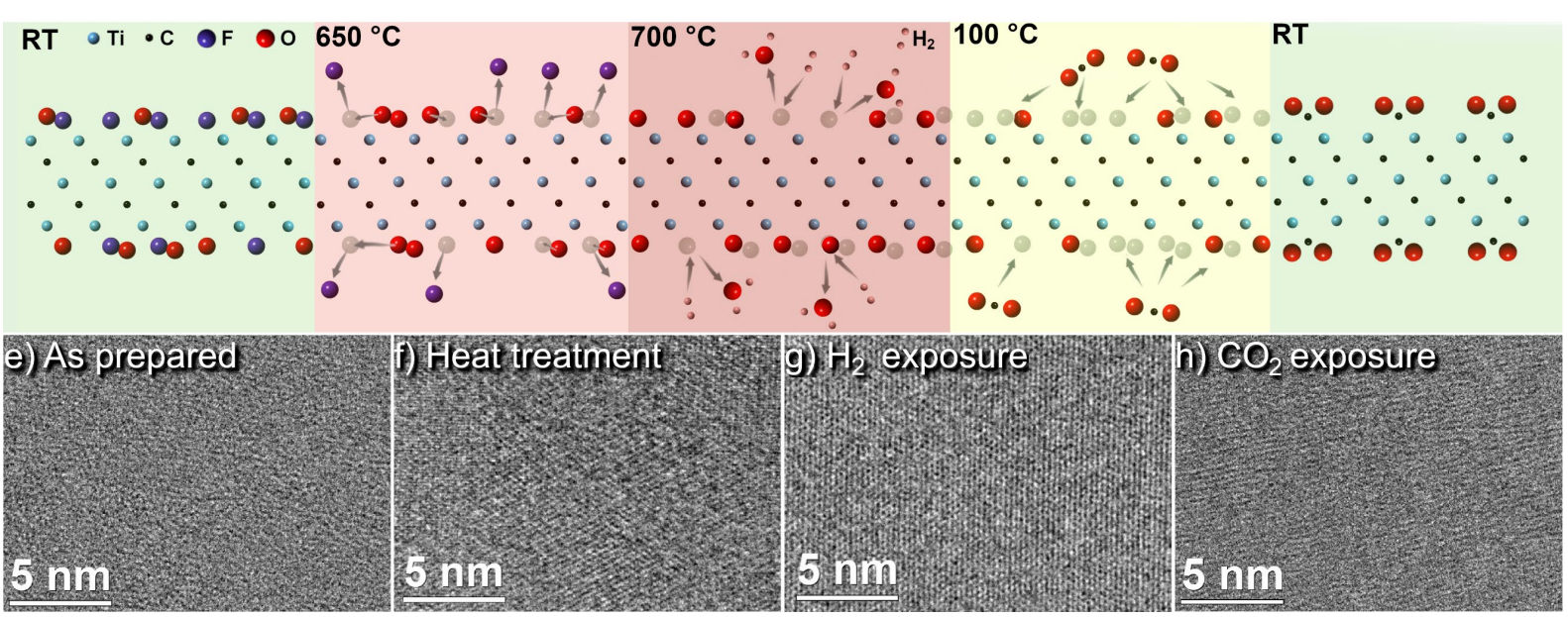

Figure 1. Cross-sectional schematics of a $\mathrm{Ti}_{3} \mathrm{C}_{2} \mathrm{~T}_{\mathrm{x}}$ flake illustrating the change in surface terminations (evolution of $\mathrm{T}_{\mathrm{x}}$ ) from left to right, as a result of thermal and environmental processing. a) As prepared, b) heat treatment, c) $\mathrm{H}_{2}$ exposure, and finally d) $\mathrm{CO}_{2}$ exposure. The corresponding plan-view TEM images after each step are presented in e-h). The images were acquired at the following conditions; e) as prepared, f) heat treatment for $12 \mathrm{~h}$ at $650{ }^{\circ} \mathrm{C}$, g) $\mathrm{H}_{2}$ exposure for $0.5 \mathrm{~h}$ at $8 \mathrm{mbar}$ and $700{ }^{\circ} \mathrm{C}$, and $\mathrm{h}$ ) $\mathrm{CO}_{2}$ exposure for $0.5 \mathrm{~h}$ at $3.5 \mathrm{mbar}$ and $100^{\circ} \mathrm{C}$. 

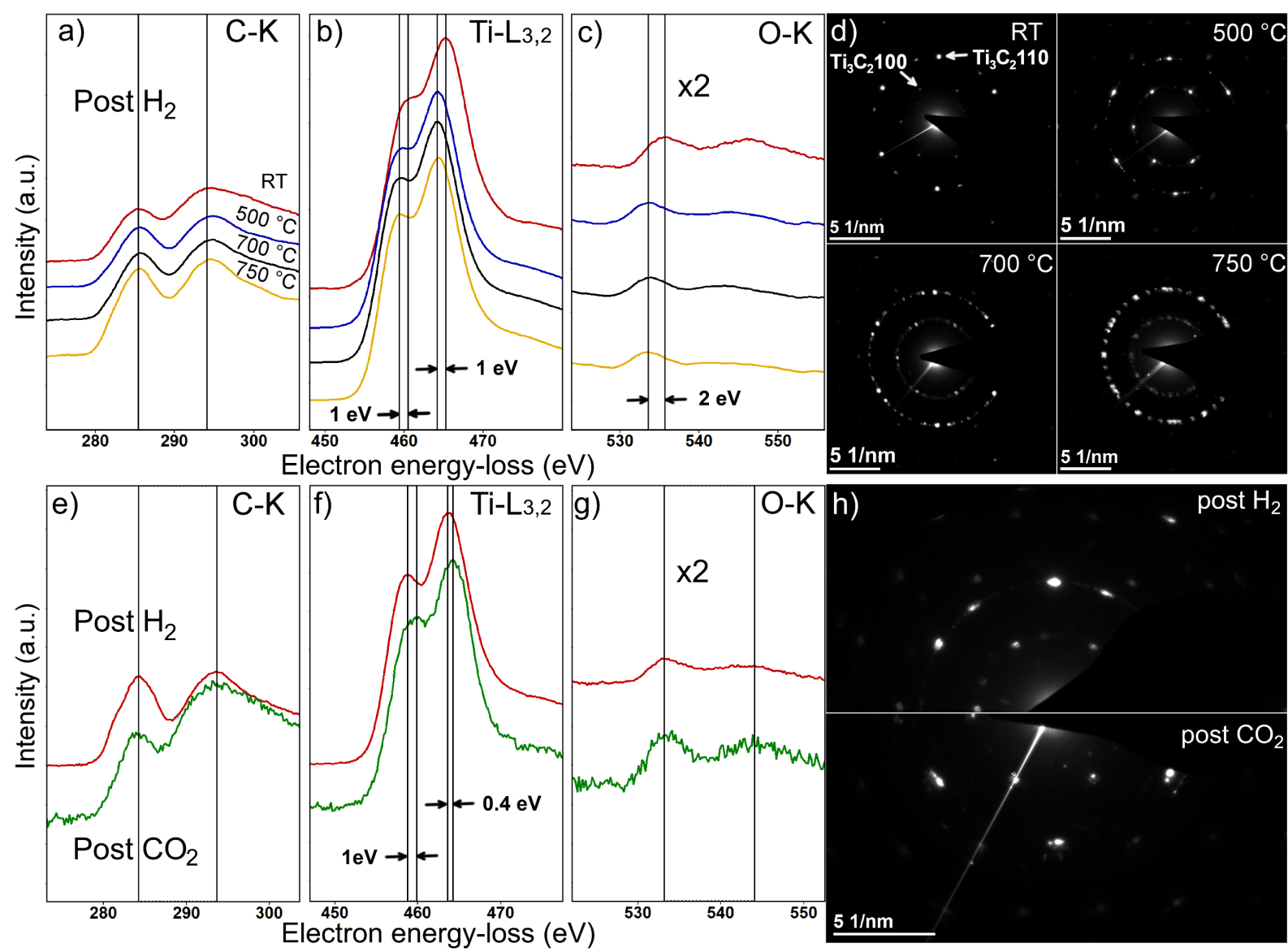

Figure 2. (a-d) EELS spectra and plan-view ED patterns acquired from $\mathrm{Ti}_{3} \mathrm{C}_{2} \mathrm{~T}_{\mathrm{x}}$ flakes at high vacuum conditions after exposure to $8 \mathrm{mbar}_{2}$ gas at $\mathrm{RT}, 500,700$, and $750{ }^{\circ} \mathrm{C}$ for $0.5 \mathrm{~h}$. (eh) EELS spectra and ED patterns acquired after exposure to $3.5 \mathrm{mbar} \mathrm{CO}_{2}$ gas at $100{ }^{\circ} \mathrm{C}$ for $0.5 \mathrm{~h}$ after a preceding $\mathrm{H}_{2}$ exposure for $1 \mathrm{~h}$ at $650{ }^{\circ} \mathrm{C}$. The EELS fine structure of C-K, Ti$\mathrm{L}_{3,2}$, and $\mathrm{O}-\mathrm{K}$ is presented. Note the $\mathrm{x} 2$ factor for $\mathrm{O}-\mathrm{K}$. 


\section{Experimental Section}

$\mathrm{Ti}_{3} \mathrm{C}_{2} \mathrm{~T}_{\mathrm{x}}$ MXene multilayer powder used in the ETEM experiments was prepared by chemical etching of $\mathrm{Ti}_{3} \mathrm{AlC}_{2}$ powder that in turn was synthesized following a procedure previously described. ${ }^{[31]}$ One gram of $\mathrm{Ti}_{3} \mathrm{C}_{2} \mathrm{~T}_{\mathrm{x}}$ powder was immersed and continuously stirred in $20 \mathrm{ml}$ aqueous solution of $\mathrm{LiF}$ and $\mathrm{HCl}$ acid with 1.5:12 molar ratio for $24 \mathrm{~h}$ at $35^{\circ} \mathrm{C}$. After etching, the mixture was washed three times each by $40 \mathrm{ml}$ of $1 \mathrm{M} \mathrm{HCl}$ to remove all the excess $\mathrm{LiF}$ salts followed by 3 cycles of washes with $40 \mathrm{ml}$ of $1 \mathrm{M} \mathrm{LiCl}$ each. The mixture was then washed with deionized (DI) water for several cycles each of $40 \mathrm{ml}$ until the supernatant reached a $\mathrm{pH}$ of approximately 6. Delamination into $(20 \mu \mathrm{m}$ x $20 \mu \mathrm{m})$ single flakes in deaerated DI-water was facilitated by gentle shaking for 10 minutes followed by centrifuging at $3500 \mathrm{rpm}$ for $1 \mathrm{~h}$. TEM samples were thereafter prepared by drop-casting $0.1 \mu$ l single-flake-solution on a DENSsolutions through hole Wildfire nanochip and placed in a DENSsolutions Wildfire singletilt heating holder. The image corrected FEI Titan ETEM at DTU equipped with a high brightness XFEG and a monochromator operated at $300 \mathrm{kV}$ achieving $\sim 0.8 \AA$ resolution was used to characterize the surfaces of single flakes. $\mathrm{H}_{2}, \mathrm{~N}_{2}$, and $\mathrm{CO}_{2}$ gas was introduced separately at temperatures ranging from room temperature up to $750{ }^{\circ} \mathrm{C}$ for pressures between $\sim 10^{-6}$ mbar -8 mbar and the samples were characterized before, during, and after exposure. EELS was acquired in diffraction mode resulting in $\sim 0 \mathrm{mrad}$ convergence angle and a $\sim 10 \mathrm{mrad}$ collection angle with a GIF Tridiem spectrometer at $\sim 1.3 \mathrm{eV}$ energy resolution and $0.2 \mathrm{eV}$ energy dispersion. EELS spectra were acquired in high vacuum conditions after gas exposure on regions that had not been subjected to the electron beam. EELS data processing was performed using the commercial Gatan Digital Micrograph software with built in routines. Quantification was done on single scattering distributions after Fourier deconvolution, powerlaw background subtraction, and employing Hartree-Slater inelastic electron scattering crosssections, excluding energy-loss near edge structures. RGA data was acquired with a Pfeiffer Vacuum mass spectrometer. HRTEM images were acquired with Gatan OneView 4K CMOS

camera. First-principle calculations were performed using DFT, see details in the supplementary information. 


\section{Acknowledgements}

The authors acknowledge the Swedish Research Council for funding under grants no. 201604412, 2016-00889 and 642-2013-8020. The Knut and Alice Wallenberg's Foundation is acknowledged for support of the electron microscopy laboratory in Linköping, a Fellowship grant and a project grant (KAW 2015.0043). The authors also acknowledge Swedish Foundation for Strategic Research (SSF) through project funding (EM16-0004) and the Research Infrastructure Fellow program no. RIF 14-0074 and no. FL12-0181. The authors finally acknowledge support from the Swedish Government Strategic Research Area in Materials Science on Functional Materials at Linköping University (Faculty Grant SFO-MatLiU No 2009 00971).

\section{Author contributions}

P.O.Å. P. conceived the research plan with input from L.-Å. N., I. P., and J. R.

The materials were prepared by J. H.

I.P. performed the experimental work and the analysis under supervision of T.W.H., J.B.W., J.P., J.R., V.D and P.O.Å.P.

H. L. performed the first-principle calculations and analysis under supervision of J.R.

The manuscript was drafted by I.P. and P.O.Å.P and finalized with input from all authors.

\section{Competing interests}

The authors declare no competing interests 


\section{References}

[1] S. Choi, J.H. Drese, C.W. Jones, Chem. Sus. Chem., 2009, 2, 796.

[2] J. Zhang, R. Singh, P.A. Webley, Micropor. Mesopor. Mater., 2008, 111, 478.

[3] C.A. Trickett, A. Helal, B.A. Al-Maythalony, Z.H. Yamani, K.E. Cordova, O.M. Yaghi, Nat. Rev. Mater., 2017, 2, 17045.

[4] X. Li, Y. Cheng, H. Zhang, S. Wang, Z. Jiang, R. Guo, and H. Wu, ACS Appl. Mater. Interfaces, 2015, 7, 5528.

[5] R. Ben-Mansour, M.A. Habib, O.E. Bamidele, M. Basha, N.A.A. Qasem, A. Peedikakkal, T. Laoui, and M. Ali, Appl. Energy, 2016, 161, 225.

[6] M. Naguib, V.N. Mochalin, M.W. Barsoum, Y. Gogotsi, Adv. Mater., 2014, 26, 992.

[7] M. Ghidiu, M.R. Lukatskaya, , M.-Q. Zhao, Y. Gogotsi, and M.W. Barsoum, Nature, 2014, 516, 78 .

[8] B. Anasori, M.R. Lukatskaya and Y. Gogotsi, Nat. Rev. Mater., 2017, 2, 16098.

[9] F. Shahzad, M. Alhabeb, C.B. Hatter, B. Anasori, S. Man Hong, C.M. Koo, Y. Gogotsi, Science 2016, 353, 1137.

[10] Q. Peng, J. Guo, Q. Zhang, J. Xiang, B. Liu, A. Zhou, R. Liu, Y. Tian, J. Am. Chem. Soc. 2014, 136, 4113.

[11] M. Naguib, M. Kurtoglu, V. Presser, J. Lu, J.J. Niu, M. Heon, L. Hultman, Y. Gogotsi and M.W. Barsoum, Adv. Mater., 2011, 23, 4248.

[12] M. Naguib, O. Mashtalir, J. Carle, V. Presser, J. Lu, L. Hultman, Y. Gogotsi and M.W. Barsoum, ACS Nano, 2012, 6, 1322.

[13] M.W. Barsoum, Prog. Solid State Chem. 2000, 28, 201.

[14] P. Srivastava, A. Mishra, H. Mizuseki, K.-R. Lee, and A.K. Singh, ACS Appl. Mater. Interfaces, 2016, 8, 24256

[15] M. Naguib, J. Halim, J. Lu, L. Hultman, Y. Gogotsi, and M.W. Barsoum, J. Amer. Chem. Soc., 2013, 135, 15966.

[16] B. Anasori, Y. Xie, M. Beidaghi, J. Lu, B.C. Hosler, L. Hultman, P.R.C. Kent, Y. Gogotsi and M.W. Barsoum, ACS Nano, 2015, 9, 9507.

[17] I. Persson, A. el Ghazaly, Q. Tao, J. Halim, S. Kota, V. Darakchieva, J. Palisaitis, M.W. Barsoum, J. Rosen, and P.O.A. Persson Small, 2018, 14, 1703676. 
[18] Q. Tao, M. Dahlqvist, J. Lu, S. Kota, R. Meshkian, J. Halim, J. Palisaitis, L. Hultman, M. W. Barsoum, P. O.Å. Persson and J. Rosen, Nat. Commun., 2017, 8, 14949.

[19] R. Meshkian, M. Dahlqvist, J. Lu, B. Wickman, J. Halim, J. Thörnberg, Q. Tao, S. Li, S. Intikhab, J. Snyder, M.W. Barsoum, M. Yildizhan, J. Palisaitis, L. Hultman, P.O.Å. Persson, and J. Rosen, Adv. Mater., 2018, 30, 1706409.

[20] M. Khazaei, A. Ranjbar, M. Arai, T. Sasaki and S. Yunoki J. Mater. Chem. C, 2017, 5,2488 .

[21] Á. Morales-García, A. Fernández-Fernández, F. Viñes, and F. Illas, J. Mater. Chem. A, 2018, 6, 3381 .

[22] N. Li, X. Chen, W.-J. Ong, D.R. MacFarlane, X. Zhao, A. K. Cheetham, and C. Sun, ACS Nano, 2017, 11, 10825.

[23] Y. Shao, F. Zhang, X. Shi, and H. Pan, Phys. Chem. Chem. Phys., 2017, 19, 28710.

[24] M.A. Hope, A.C. Forse, K.J. Griffith, M.R. Lukatskaya, M. Ghidiu, Y. Gogotsi, and C.P. Grey, Phys. Chem. Chem. Phys., 2016, 18, 5099.

[25] I. Persson, L.-Å. Näslund, J. Halim, M.W. Barsoum, J. Palisaitis, V. Darakchieva, J. Rosen, and P.O.Å. Persson, 2D Materials, 2017, 5, 015002.

[26] M. Khazaei, M. Arai, T. Sasaki, C.-Y. Chung, N.S. Venkataramanan, M. Estili, Y. Sakka, and Y. Kawazoe, Adv. Funct. Mater., 2013, 23, 2185.

[27] L.H. Karlsson, J. Birch, J. Halim, M.W. Barsoum, and P.O.Å. Persson, Nano Lett., 2015, 15, 4955 .

[28] X. Sang, Y. Xie, D.E. Yilmaz, R. Lotfi, M. Alhabeb, A. Ostadhossein, B. Anasori, W. Sun, X. Li, K. Xiao, P.R.C. Kent, A.C.T. van Duin, Y. Gogotsi and R.R. Unocic, Nature Commun., 2018, 9, 2266.

[29] Z. R. Herm, J. A. Swisher, B. Smit, R. Krishna, and J. R. Long, J. Am. Chem. Soc., 2011, 133, 5664.

[30] S. Cavenati, C. A. Grande, and A. E. Rodrigues, J. Chem. Eng. Data, 2004, 49, 1095.

[31] A. Miranda, J. Halim, M.W. Barsoum, A. Lorke. Appl. Phys. Lett., 2016, 108, 033102. 